(Aus dem Kinderasyl der Stadt Berlin [Oberarzt: Prof. Dr. H. Finkelstein].)

\title{
Zur Wirkung der Kohlehydrate auf den Anwuchs im Säuglingsalter. ${ }^{1}$ )
}

\author{
Von \\ Dr. Iwan Rosenstern, \\ Assistenten. \\ Mit 8 Textabbildungen.
}

(Eingegangen am 9. Juli 1914.)

Die heute geübte Behandlung der Ernährungsstöringen im Säuglingsalter ist im wesentlichen eine Nährstofftherapie. Sie besteht in einer Ausschaltung bzw. Reduktion gewisser Nährstoffe, in der Anreicherung anderer. Ihre Grundlage bildet die Erkenntnis, daß zwischen Ernährungsstörung auf der einen, Quantität und Qualität der Nahrung auf der anderen Seite sehr nahe Beziehungen bestehen. Die Arbeit der letzten Jahre auf diesem Gebiete hat der Erforschung und dem weiteren Ausban dieser Beziehungen gegolten und das Ergebnis gezeitigt, daß heute für das ernährungsgestörte Kind joder Nährstoff seine bestimmte Note hat, verschieden allerdings nach der Art der Störung und abhängig von der Zusammensetzung der Nahrung, innerhalb deren Verband der Stoff gereicht wird. Die energetische Betrachtungsweise ist dabei in den Hintergrund getreten.

Anders beim ges unden Kind. Hier steht das energetische Prinzip noch im Vordergrunde, und der einzelne Nährstoff findet, z. B. bei der Aufstellung neuer Normalnahrungen nur insoweit Berücksichtigung, als es sich darum handelt, Korrelationen zu schaffen, bei denen Störungen nach Möglichkeit vermieden werden. Stillschweigend wird dabei so viel von den einzelnen notwendigen Nährstoffen gegeben, daß der Bedarf gedeckt ist.

1) Bezüglich der Literatur vgl. die zusammenfassenden Darstellungen von Czerny-Keller, Lehrbuch; Klotz, Ergebnisse d. i. Med. u. Kinderheilk.; Calvary, Egebnisse d. i. Med. u. Kinderheilk. 
Bei dieser Betrachtungsweise ist ein Problem momentan in den Hintergrund getreten, das Problem von der spezifischen Funktion der einzelnen Nährstoffe für den gesunden wachsenden Organismus, d. h. von der Funktion, die über den Wert des Nahrungsstoffes als Brennmaterial hinausgeht. Viel diskutiert ist in dieser Hinsicht bereits die Rolle des Eiweißes, weniger die des Zuckers und des Fettes.

$\mathrm{DaB}$ die letztgenannten Stoffe neben ihrer rein energetischen Bedeutung spezifische Wirkungen entfalten, zeigt die klinische Erfahrung immer wieder. Wenn wir beispielsweise in der Nahrung plötzlich das Fett soweit dies möglich durch isodyname Mengen Zucker ersetzen oder umgekehrt, so treten ganz erhebliche Veränderungen auf und zwar gesetzmäßige als Ausdruck der spezifischen Leistung der Stoffe.

Hier zunächst einige Worte über den Begriff der spezifischen Funktion. Spezifisch im engeren Sinne des Wortes wird man diejenigen Leistungen nennen müssen, die von anderen Nährstoffen, auch wenn diese in noch so großen Mengen verabreicht werden, nicht geleistet werden können. Wenn z. B. ein Minimum von Zucker in der Nahrung erforderlich ist für eine normale $\mathrm{N}$-Retention, so handelt es sich hier um eine ,absolut spezifische" Leistung. Im weiteren Sinne des Wortes könnte man aber alle diejenigen Wirkungen eines Nährstoffes spezifisch nènnen, die in isody namen Mengen von anderen Nährstoffen nicht geleistet werden können. Wenn z. B. ein gewisses Minimum von Zucker in der Nahrung vorhanden ist, so werden darüber hinaus Fett und Zucker eine stickstoffsparende Wirkung entfalten, aber das Fett erst in weit größeren Mengen als isodyname Mengen Zucker.

Ähnliches ergibt sich, wenn wir die Wirkung von Fett und Zucker auf den Verlauf der Körpergewichtskurve beim gesunden wachsenden Säugling betrachten. Unterhalb eines gewissen Minimums von Zucker ist überhaupt ein Gewichtswachstum nicht möglich, auch dann nicht, wenn wir noch so große Mengen an sonstigem Nährmaterial verabreichen. Ist dies absolute Zuckerminimum aber in der Nahrung vorhanden, so läßt sich, sowohl mit Zucker als auch mit Fett ein Gewichtsansatz erzielen, allerdings nur durch Fettmengen, die den Energiegehalt der den gleichen Effekt erreichenden Zuckerquantitäten um ein erhebliches überschreitet.

Man könnte so von einer absolut und relativ spezifischen Wirkung der Nährstoffe sprechen. In praxi wird es nicht möglich sein, immer scharf zwischen diesen beiden Arten der Wirkung zu unterscheiden. 
Am meisten wissen wir über die spezifischen Wirkungen der spezifischen Funktionen des Zuckers. Seit langem bekannt ist die Bedeutung des Zuckers für die normale Fettverbrennung, für die Wärmeregulation, für den $\mathrm{N}$-Stoffwechsel. Wichtiger noch als diese Beziehungen erscheint für den wachsenden Organismus die Wechselwirkung zwischen Zucker und Wasserretention, auf die zuerst Czern y und Fre und hingewiesen haben. Die tägliche Erfahrung erweist sie aufs neue: ohne eine gewisse Menge Zucker ist auch bei Deckung des Energiebedarfs, wie schon erwähnt, eine Körpergewichtszunahme unmöglich, und diese beruht nun mal zum größeren Teile auf Wasseransatz; durch Superposition von Zucker gelingt es, beim Gesunden so gut wie immer, einen steilen Aufschwung der Gewichtskurve zu erzeugen, der nur durch Wasserretention erklärt werden kann; und endlich findet bei Entziehung des vorher reichlich verabreichten Kohlehydrats unter Umständen ein starker Gewichtssturz statt, der wiederum hauptsächlich in einer Wasserausschwemmung bestehen muB.

In welcher Weise der Zucker wasserbindend wirken kann, dafür kommen verschiedene Möglichkeiten in Frage. Zunächst die, daß der Zucker in Form von Glykogen deponiert wird, was bekanntlich unter Bindung von 3 Teilen Wasser geschieht. Es könnten weiter die osmotischen Eigenschaften des Zuckers in Betracht kommen (Schloß3). Bei dicser Annahme müßte der Zucker zum mindesten für einige Zeit als solcher unverbrannt im Organismus kreisen, so daß ganz ähnliche Verhältnisse entstehen wie bei der Salzretention. Aber beide Möglichkeiten reichen, wie ich früher gezeigt habe, nicht aus, um die in extremen Fällen zi beobachtende maximale Zunahme bei minimalen Mengen von Zuckerzulage zu erklären (bei $5 \mathrm{~g}$ Zuckerzulage pro die imnerhalb 6 Tagen $420 \mathrm{~g}$ Zunahme, d. h. $60 \mathrm{~g}$ pro die). Es kan n also nur eine indirekte Wirkung vorliegen. Das alles lehrt uns die Klinik.

Uber den Mechanismus dieser indirekten Zuckerwirkung können uns die Stoffwechselversuche orientieren. Es liegen eine ganze Anzahl solcher Untersuchungen vor, die ergeben, daß unter Zuckerzulage eine Verbesserung der $\mathrm{N}$-, Cl- und Na-Bilanz auftritt, und daß bei /uckerentziehung ein Umschlagen der vorher stark positiven $\mathrm{N}$-, Cl- und Aschebilanz in eine negative stattfindet (vgl. z. B. die neuesten Untersuchungen von Raoul y Carneiro). Geringfügiger sind unsere Kenntnisse über die Wirkung der Zuckerzulage auf die Bilanz der alkalischen Erden. Hier liegen die Ergebnisse von Birk ${ }^{1}$ ) und Rothberg ${ }^{2}$ ) vor,

1) Birk, Jahrb. f. Kinderheilk. 66. 2) Roth berg, Jahrb. f. Kinderheilk. 66. 
die eine Versehlechterung der Kalkbilanz bei kohlehydratreicher Nahrung zeigen, weiterhin die Untersuchungen von Fre und ${ }^{1}$ ), der durch Malzzugabe beim rachitischen Kind eine Steigerung der Kalkbilanz erzielte, die aber beim normalen ausblieb. Und endlich hat Kochma. $n n^{2}$ ) jüngst an gesunden ausgewachsenen Hunden nachgewiesen, daß Kohlehydrate (ebenso-wie Eiweiß und Fett) die Kalkbilanz in der Weise beeinflussen, daß ihre Zulage zu der ursprünglichen Nahrung das vorher bestehende Kalkgleichgewicht stört und den Organismus zur Abgabe erheblicher Kalkmenge zwingt.

Was an Stoffwechselresultaten über die Bilanz der alkalischen Erden bei Zuckerzulage vorliegt, ist zum Teil widersprechend; die Resultate über die Stickstoff-, Chlor und Aschebilanz sind zum großen Teil bei einer Versuchsanordnung gewonnen, die nicht rein ist. Vielfach war in der Vorperiode der Nahrungsbedarf nicht gedeckt, in anderen Fällen wurde zugleich mit der Zuckerzulage die Zusammensetzung der Versuchsnahrung auch sonst erheblich geändert.

Um über den Mechanismus der Zuckerwirkung ins klare zu kommen, haben wir deshalb nochmals zwei Versuchsreihen vorgenommen. Die Fragestellung lautete: Wie verhält sich der Stoffwechsel des gesunden Kindes, wenn bei Deckung des Energiebedarfs und Zufuhr der notwendigen Wasser-, Salz-, Fett- und Eiweißmengen der Zuckergehalt der Nahrung unter das zur Körpergewichtszunahme erforderliche Minimum sinkt? Welche Veränderungen treten weiter bei Zuckerzulage ein?

Die Versuchsanlage konnte dabei eine verschiedene sein. Man konnte einmal eine bestimmte Menge von Fett, das in der Vorperiode zur Deckung des Bedarfs in größerer Menge verabreicht werden mußte, durch Zucker ersetzen. Dann wären die Bedingungen insofern gleichgeblieben, als die Energiezufuhr sich nicht änderte. Man hätte die so erhaltenen Resultate aber ebensogut auf Fettreduktion, wie auf Zuckerzulage zurückführen können. Und gerade mit Rücksicht auf die strittigen Beziehungen zwischen Fett und Kalk beim gesunden Organismus wären hier die Resultate sehr diskutabel gewesen. Um diese Schwierigkeit zu vermeiden, wurde ein anderer Weg eingeschlagen, d. h. der Zucker superponiert. Dabei änderte sich allerdings die Energiezufuhr, aber da der Bedarf vorher gedeckt war, erschien uns diese Art des Vorgehens geeigneter.

Der Prüfung unterzogen wurde der Stickstoff-, Fett-, Kalk-, Phosphor-, Natrium-, Kalium- und Chlorstoffwechsel. Die Anlage des

1) Fre und, Ergebnisse d. i. Med. u. Kinderheilk. 3; Biochem. Zeitschr. 1909.

2) Kochmann und Petzsch, Biochem. Zeitschr. 3?. 
Zur Wirkung der Kohlehydrate auf den Anwuchs in Sáuglingsalter. 337

Versuchs war derart, daß zunächst für eine Zeitlang Eiweißmilch mit Zulage von salzfreier Butter in einer Menge verabreicht wurde, die den Energiebedairf deckte (100 Cal, pro Kilogramm Körpergewicht). Daß bei dieser Nahrung auch der Bedarf an sonstigen notwendigen Stoffen (Eiweiß, Salze, Wasser) befriedigt war, werden die. folgenden Zahlen lehren. Infolge des geringen Zuckergehaltes der Nahrung verblieb das Gewicht in der Horizontalen. Nach einer gewissen Beobachtungsdauer wurde nun ein Stoffwechselversuch vorgenommen (Vorperiode). In der folgenden Hauptperiode wurde Zucker zugelegt, dabei starker Gewichtsansatz, und in der Nachperiode wurde der Zucker wieder eliminiert.

Ich bemerke, daß die Untersuchungen an Kindern vorgenommen wurden, die sich sowohl früher wie später als völlig frei von irgendwelchen Krankheitserscheinungen erwiesen, und daß der Durchführung der Untersuchungen durch das Verhalten der Kinder keinerlei Sehwierigkeiten erwuchs. Die Kinder fühlten sich auf der Schwebe wohl, es erfolgte kein einziges Mal Speien oder Erbrechen. Die Stühle blieben in ihrer Konsistenz vollkommen unverändert.

Bezüglich der Methodik verweise ich nuf die früheren Mitteilıngen von L. F. Meyer.

\section{Versuch 1.}

Kind K. R. (Kurve 1), geb. 30. VIII. 1912. Aufnahme 9. IX. 1912. Gut genährtes, rosiges Kind mit gutem lettpolster und kräftigem Knochenbau. Aufnahmegewicht $3700 \mathrm{~g}$. Zunächst Ernährung mit Eiweißmilch und 5\% Zuckerzusatz. Dabei gutes Gedeihen. Regelmäßige Gewichtszunahme. Konsistente braune Stühle. Es erfolgt alsdann Ersatz des Zuckerzusatzes durch annähernd isodyname Mengen salzfreier Butter (2 proz.). Sofort Gewichtsstillstand, anch bei Steigerung der Nahrungsmenge. Die Nahrung enthält nunmehr bei einem Gehalt von $24 \mathrm{~g}$ Eiweiß 37,7 g Fett und 12,6 g Kohlehydate, eine Energiemenge von 500 Calorien. Das Kind erhält also 100 Calorien pro Kilogramm. Nunmehr Zulage von 40.g Milchzucker, sofort Ansteigen der Gewichtskurve und Zunahme von $330 \mathrm{~g}$ in 4 Tagen. Bei Entziehung des Zuckers Sinken der Kurve, die dann auf gleicher Höhe verbleibt.

Die Anlage des. Versuchs erhellt aus Kurve 1.

Der Versuch gliedert sich -in 5 Perioden. Die Grundnahrung besteht in allen 5 Perioden aus $840 \mathrm{~g}$ Eiweißmilch plus $2 \%$ Butter. Dazu addiert sich in den Hauptperioden $(2+3) \mathbf{4 0} \mathrm{g}$ Milehzucker pro die. 
Ich bemerke, daß die Stühle während der ganzen Versuchszeit von fester Konsistenz waren und eine hellgelbe Farbe hatten, die nur während der Zeit der Zuckerzugabe einem etwas dunkleren Tone Platz machte.

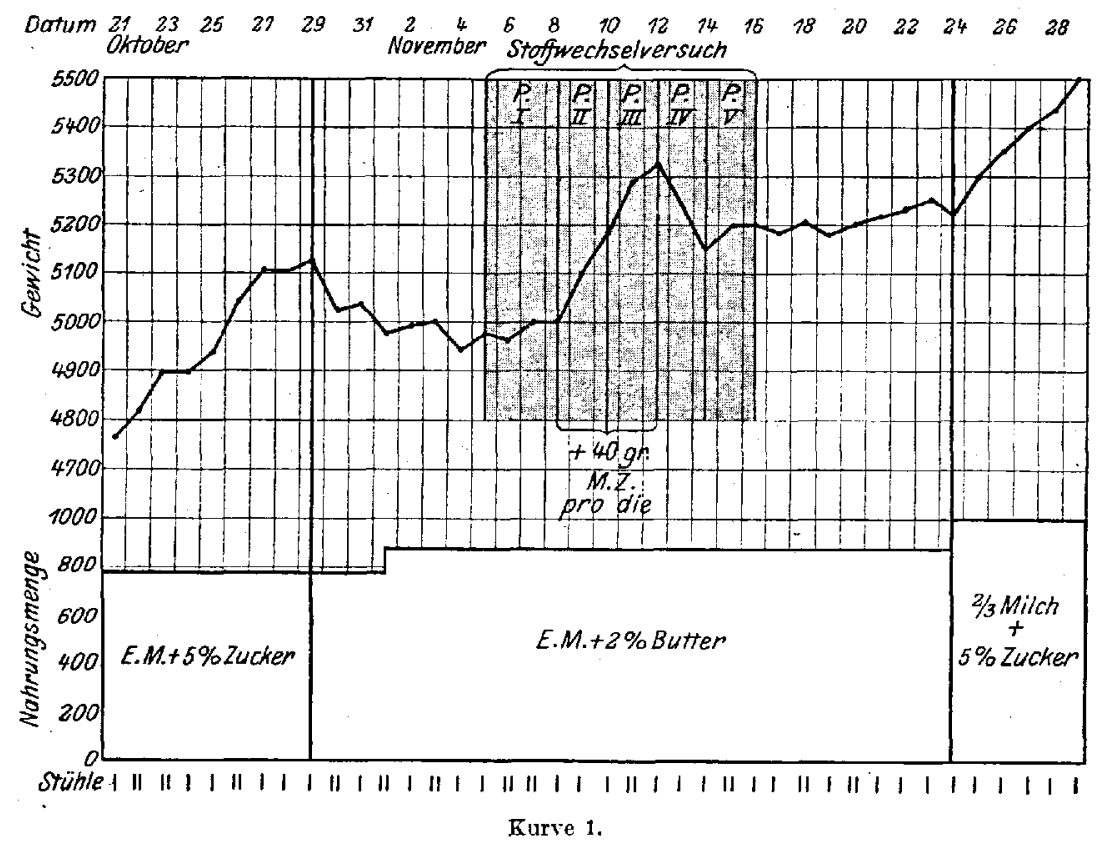

Die Resultate seien der Übersicht halber gleich in Tabellenform gegeben, und zwar alle Werte auf den Tag berechnet.

Gesamitettbilanz (pro die).

\begin{tabular}{|c|c|c|c|c|c|}
\hline \multirow{2}{*}{ Periode } & \multirow{2}{*}{ Nahrung } & \multirow{2}{*}{ Fetteiniuhr } & \multirow{2}{*}{$\begin{array}{l}\text { Gesamtiett } \\
\text { im Kot }\end{array}$} & \multicolumn{2}{|c|}{ Fettresorption } \\
\hline & & & & absolut & $\mid \begin{array}{l}\% \text { der Fin- } \\
\text { fuhr }\end{array}$ \\
\hline I & $840 \mathrm{~g}$ Eiweißmilch $+2 \%$ Butter & 87,72 & 0,90 & 36,82 & 97,9 \\
\hline I! & $\begin{array}{c}840 \mathrm{~g} \text { Eiweifmilch }+2 \% \text { Butter } \\
-40 \mathrm{~g} \text { Milchzucker }\end{array}$ & 37,72 & 1,24 & 36,48 & 96.7 \\
\hline III & do. & 37,72 & 1,07 & 36,60 & 97,2 \\
\hline IV & $840 \mathrm{~g}$ Eiwoißmileh $+2 \%$ Butter & 37,72 & 1,16 & 36,56 & 96,9 \\
\hline $\mathrm{V}$ & do. & 37,72 & 1,03 & 36,69 & 96,5 \\
\hline
\end{tabular}

Man sieht, daß die Fettausnutzung eine ausgezeichnete ist. Die Resorption beträgt zwischen 96,5 und $97,9 \%$. Durch die Zuckerzulage wird die Gesamtresorption nicht verändert. 
Fettverteilung im Kot.

\begin{tabular}{|c|c|c|c|c|c|c|}
\hline Periode & Nahrung & $\begin{array}{l}\text { Gesamt- } \\
\text { fett im } \\
\text { Kot }\end{array}$ & $\begin{array}{c}\text { Neutral- } \\
\text { fett } \\
+ \text { Fett- } \\
\text { säuren } \\
\text { a bsolut }\end{array}$ & $\begin{array}{l}\text { Fettsei- } \\
\text { fen ab- } \\
\text { solut }\end{array}$ & $\begin{array}{c}\text { Neutral- } \\
\text { fett } \\
+ \text { Fett- } \\
\text { süuren } \\
\% \\
\end{array}$ & $\begin{array}{l}\text { Fettsei- } \\
\text { fen } \%\end{array}$ \\
\hline $\mathrm{I}$ & $840 \mathrm{~g}$ Eiweißmilch $+2 \%$ Butter & 0,90 & 0,51 & 0,39 & 56,6 & 43,4 \\
\hline II & $\begin{array}{c}840 \text { g Eiweißmilch }+2 \% \text { Butter } \\
+40 \mathrm{~g} \text { Milchzucker }\end{array}$ & 1,24 & 0,88 & 0,36 & 70,9 & 89,1 \\
\hline III & $=$ do: & 1,07 & 0,78 & 0,29 & 72,9 & 27,1 \\
\hline IV & $840 \mathrm{~g}$ Eiweißnilch $+2 \%$ Butter & 1,16 & 0,85 & 0,31 & 73,3 & 26,7 \\
\hline $\mathrm{V}$ & do. & 1,03 & 0,85 & 0,18 & 82,5 & 17,5 \\
\hline
\end{tabular}

Bezüglich der Fettverteilung sind die erhaltenen Werte infolge ihrer geringen absoluten Menge nur mit Vorsicht zu bewerten. Auffallend ist immerhin der hohe Prozentsatz an Seifen. Die Seifenbildung wird durch die Zuckerzulage kaum beeinflußt. Wenn ihr prozentualer Anteil in der zweiten und dritten Periode etwas sinkt, so ist das im wesentlichen auf eine leichte Erhöhung der Neutralfettansscheidung $z u$ beziehen. Sie ist aber um so weniger zu verwerten, als auch in der Periode 4 und 5 der Prozentsatz an Seifen weiter sinkt.

N-Bilanz (pro die).

\begin{tabular}{|c|c|c|c|c|c|c|c|}
\hline \multirow{2}{*}{$\begin{array}{l}\text { Pe- } \\
\text { riode }\end{array}$} & \multirow[b]{2}{*}{ Nahrung } & \multirow[b]{2}{*}{ Einfuhr } & \multicolumn{2}{|c|}{ Kot } & \multirow[b]{2}{*}{ Urin } & \multicolumn{2}{|c|}{ Retention. } \\
\hline & & & absolut & $\begin{array}{l}\text { In \% der } \\
\text { Einfuhr }\end{array}$ & & absolut & $\begin{array}{l}\% \text { der } \\
\text { Finfuhr }\end{array}$ \\
\hline I & $840 \mathrm{~g}$ Eiweißmilch $+2 \%$ Butter & 3,763 & 0,236 & 6,28 & 3,629 & $-0,102$ & \\
\hline II & $\begin{array}{l}840 \mathrm{~g} \text { Eiweilmilch }+2 \% \text { Butter } \\
+40 \mathrm{~g} \mathrm{Milchzucker}\end{array}$ & 3,763 & 0,276 & $7,3 \pm$ & 3,076 & +0.411 & 10,92 \\
\hline III & do. & 3,763 & 0,275 & 7,30 & 3,004 & $+10,481$ & 12,06 \\
\hline IV & $840 \mathrm{~g}$ Eiweißmilch of $2 \%$ Butter & 3,763 & 0,335 & 8,90 & 3,466 & $-0,038$ & \\
\hline $\mathrm{V}$ & do. & 8,763 & 0,314 & 8,34 & 3.577 & +0.128 & \\
\hline
\end{tabular}

Sehr auffallend ist die N-Bilanz. Trotz der sehr hohen Eiweißzufuhr ist sie in den Perioden I, IV und. V eine negative, um mit der Zuckerzufuhr positive Werte zu erreichen. Dabei ist die Ausnutzung in allen 5 Perioden eine gute.

Was das Verhalteri der N-Bilanz zum Gewicht betrifft, so gehen beide Werte nicht ganz parallel. Periode $I$ und $V$ zeigen eine negative Bilanz bei zunehmendem Gewicht, und die Retention in den Perioden II und III entspricht nicht annähernd der starken Zunahwe des Gewichts $(0,9 \mathrm{~N}$-Retention bei $300 \mathrm{~g}$ Gewichtszunahme). 
$\mathrm{Na}_{2} \mathrm{O}$-Bilanz (prodie).

\begin{tabular}{|c|c|c|c|c|c|c|c|}
\hline \multirow{2}{*}{$\begin{array}{c}\text { Pe- } \\
\text { riode }\end{array}$} & \multirow{2}{*}{ Fahrung } & \multirow[b]{2}{*}{ afuhr } & \multicolumn{2}{|c|}{ Kot } & \multirow{2}{*}{ Urin } & \multicolumn{2}{|c|}{ Retention } \\
\hline & & & absolut & $\begin{array}{l}\text { in \% der } \\
\text { Einfuhr }\end{array}$ & & absolut & $\begin{array}{l}\text { \%inder } \\
\text { Einfuhr }\end{array}$ \\
\hline I & $840 \mathrm{~g}$ Eiweißmilch $+2 \%$ Butter & 0,309 & $0,030 \uparrow$ & 9,71 & 0,212 & $+0,06 \gamma$ & 21,68 \\
\hline II & $\begin{array}{l}840 \mathrm{~g} \text { Fiweißmilch }+2 \% \text { Butter } \\
\quad+40 \mathrm{~g} \text { Milchzucker }\end{array}$ & 0,309 & 0,029 & 9,39 & 0,091 & $+0,189$ & 61,17 \\
\hline III & do & 0,309 & 0,046 & 14,89 & 0,149 & $+0,114$ & 36,89 \\
\hline IV & $840 \mathrm{~g}$ Eiweißmil $h+2 \%$ Hutter & 0,309 & 0,050 & 16,18 & 0,419 & $-0,160$ & \\
\hline$V$ & do & 0,309 & 0,025 & 8,09 & 0,182 & $+0,102$ & 33,01 \\
\hline
\end{tabular}

Die Na-Bilanz in Periode I mäßig positiv wird durch die Zuckerzulage eine stärkere, um mit der Entziehung des Zuckers in Periode IV wieder zu negativen Werten zu gelangen. Die Retentionswerte in II und III sind im Verhältnis zur Gewichtszunahme gering.

$\mathrm{K}_{2} \mathrm{O}-\mathrm{Bilanz}$ (pro die).

\begin{tabular}{|c|c|c|c|c|c|c|c|}
\hline $\begin{array}{l}\text { Pe- } \\
\text { riode }\end{array}$ & Nahrung & Einfuhr & absolut & $\begin{array}{l}\text { Cot } \\
\text { in \% der } \\
\text { Einfuhr }\end{array}$ & Urin & $\begin{array}{r}\text { Rete } \\
\text { absolut }\end{array}$ & $\begin{array}{l}\text { ntion } \\
\text { in \% der } \\
\text { Einfuhr }\end{array}$ \\
\hline I & $840 \mathrm{~g}$ Eiweißmileh $+2 \%$ Butter & 0,670 & 0,072 & 10,75 & 0,640 & $-0,042$ & \\
\hline II & $\begin{array}{l}840 \mathrm{~g} \text { Eiweißmilch }+2 \% \text { Butter } \\
\quad+40 \mathrm{~g} \mathrm{Milchzucker}\end{array}$ & 0,670 & 0,095 & 14,18 & 0,469 & $+0,106$ & 15,82 \\
\hline III & do. & 0,670 & 0,047 & 7,01 & 0,503 & $+0,120$ & 17,91 \\
\hline IV & $840 \mathrm{~g}$ liweißmileh $+2 \%$ Butter & 0,670 & 0,075 & 11,20 & 0,681 & $-0,086$ & \\
\hline$r$ & do. & 0,670 & 0,099 & 14,78 & 0,458 & $+0,113$ & 16,87 \\
\hline
\end{tabular}

Die $\mathrm{K}_{2} \mathrm{O}$-Bilanz verhält sich ganż ähnlich der $\mathrm{Na}_{2} \mathrm{O}$-Bilanz.

Cl(ClNa)-Bilanz (pro`die)

\begin{tabular}{|c|c|c|c|c|c|c|c|}
\hline \multirow{2}{*}{$\begin{array}{c}\text { Po- } \\
\text { riode }\end{array}$} & \multirow[b]{2}{*}{ Nahrung } & \multirow[b]{2}{*}{ Einfuhr } & \multicolumn{2}{|c|}{ Kot } & \multirow[b]{2}{*}{ Urin } & \multicolumn{2}{|c|}{ Retention } \\
\hline & & & absolut & {$\left[\begin{array}{l}\text { is } \% \text { der } \\
\text { Ginfuhr }\end{array}\right.$} & & absolut & $\begin{array}{l}\text { in } \% \text { d d } \\
\text { Einfuhr }\end{array}$ \\
\hline I & $840 \mathrm{~g}$ Eiweißnileh $+2 \%$ Butter & 0,500 & 0,005 & 1,00 & 0,570 & $-0,075$ & \\
\hline II & $\begin{array}{c}840 \mathrm{~g} \text { Eiweißmilch }+2 \% \text { Butter } \\
40 \mathrm{~g} \text { Milchzucker }\end{array}$ & 0,500 & 0,015 & 3,33 & 0,425 & $+0,060$ & 12,00 \\
\hline III & do. & 0,500 & 0,025 & 5,00 & 0,455 & $+0,020$ & 4,00 \\
\hline IV & $840 \%$ Eiweilmilch $+2 \%$ Butter & 0,500 & 0,033 & 6,60 & 0,980 & $-0,513$ & \\
\hline $\mathbf{V}$ & do. & 0,500 & 0,039 & 7,80 & 0,543 & $-0,082$ & \\
\hline
\end{tabular}

Die Chlorbilanz ist in Periode I, IV und V negativ und wird durch die Zuckerzulage auch nur leicht positiv. Am stärksten sind die Verluste bei der plötzlichen Entziehung des Zuckers. Eine absolute Parallele zu dem Verhalten des Gewichts ist auch hier nicht zu konstatieren: Negative Werte in Periode $I$ und $V$ bei Gewichtszunahme. 
CaO-Bilanz (pro die).

\begin{tabular}{|c|c|c|c|c|c|c|c|}
\hline $\begin{array}{c}\text { Pe- } \\
\text { riode }\end{array}$ & Nahrung & Einfuhr & absolut & $\begin{array}{l}\text { Kot } \\
\text { |in \% der } \\
\text { Einfuhr }\end{array}$ & Urin & $\begin{array}{r}\text { Reter } \\
\text { absolut }\end{array}$ & $\begin{array}{l}\text { in \% der } \\
\text { Einfuhr }\end{array}$ \\
\hline I & $840 \mathrm{gr}$ & 1,219 & 0,717 . & 58,82 & & $+0,502$ & 41,18 \\
\hline II & $\begin{array}{c}840 \mathrm{~g} \text { Eiweißmilch }+2 \% \text { Butter } \\
+40 \mathrm{~g} \text { Milchzucker }\end{array}$ & 1,219 & 0,728 & 59,72 & & +10.491 & 40,28 \\
\hline III & do. & 1,219 & 0,697 & 57,18 & & $+0,522$ & 42,82 \\
\hline IV & $840 \mathrm{~g}$ Eiweißmilch $+2 \%$ Butter & 1.219 & 0,812 & 66,61 & 0,039 & $+0,368$ & 30,19 \\
\hline $\mathrm{V}$ & do. & 1,219 & 0,760 & 62,35 & & $+0,459$ & 37,65 \\
\hline
\end{tabular}

Im Gegensatz zu den bisher besprochenen Bilanzen steht das Verhalten des Kalks. Auch in der Vorperiode gut retiniert bleiben seine Werte durch Zuckerzulage unbeeinflußt, nur in Periode IV bei plötzlicher Reduktion des Zuekers ist ein leichtes Sinken der Retentionswerte zu bemerken.

$-\mathrm{P}_{2} \mathrm{O}_{5}-$ Bilanz (pro die).

\begin{tabular}{|c|c|c|c|c|c|c|c|}
\hline $\begin{array}{c}\text { Pe- } \\
\text { riode }\end{array}$ & Nahrung & Einfuhr & $\begin{array}{r}K \\
\text { absolut }\end{array}$ & $\begin{array}{l}\text { ot } \\
\text { in \% der } \\
\text { Einfuhr }\end{array}$ & Urin & $\begin{array}{l}\text { Reten } \\
\text { absolut }\end{array}$ & $\begin{array}{l}\text { in \% der } \\
\text { Einfuls }\end{array}$ \\
\hline$I$ & $840 \mathrm{~g}$ Eiweißmilch $+2 \%$ Butter & 1,157 & 0,287 & 24,81 & 0,533 & $+0,333$ & 29,13 \\
\hline II & $\begin{array}{c}840 \mathrm{~g} \text { Eiweißmilch }+2 \% \text { Butter } \\
+40 \mathrm{~g} \text { Milch zucker }\end{array}$ & 1,157 & 0,281 & 24,29 & 0,642 & $+0,234$ & 20,22 \\
\hline III & do. & 1,157 & 0,266 & 22,99 & 0,561 & $+0,330$ & 28,52 \\
\hline IT & $840 \mathrm{~g}$ EiweiBnilch $+2 \%$ Butter & 1,157 & 0,367 & 31,72 & 0,754 & $+0,036$ & 3,11 \\
\hline 1 & do. & 1,157 & 0,321 & 27,66 & 0,444 & $+0,392$ & 33,88 \\
\hline
\end{tabular}

Ganz ähnlich der Kalkbilanz verhält sich die Phosphorbilanz: Von vornherein gute Retention, durch Zuckerzulage keine Veränderung, bei plötzlicher Entziehung des Zuckers Abfallen des Wertes (Periode IV). sodann Rückkehr zu der ursprünglichen Höhe.

\section{Versuch II.}

Kind Kurt W., geb. 2. II. 19!3 (vgl. Kurve 2). Aufnahme 22. II. 1913. Gewicht bei der Aufnahme: $3700 \mathrm{~g}$. Etwas blasses, aber gut genährtes Kind. Am Hinterkopf einige kleine Furunkel. Sonst keinerlei Krankheitserscheinungen. Ernährung mit gelabter Vollmilch. Dabei leicht dyspeptische Entleerungen, langsame Gewichtszunahme. Von der 6. Woche an Eiweißmilch mit 5\% Zucker. Bei der letztgenannten Ernährung befriedigende Entwicklung. Bis zum 5. V. Gewichtszunahme auf $5000 \mathrm{~g}$, Körperlänge $58^{3} / 4 \mathrm{~cm}$. Nunmehr Ubergang auf Eiweißmilch mit $2 \%$ Butterzulage. Sofort biegt die bis dahin aufsteigende 
Gewichtskurve um, fast bis zur Horizontalen. An Stelle der vorher mehr. gelblich gefärbten Stühle treten graue bis weißliche feste En'tleerungen. Bis zum 14. VII. - hier setzt die Kurve ein - nimmt das Gewicht bei der zuckerarmen und fettreichen Ernährung um $500 \mathrm{~g} \mathrm{zu}$, d. h. $50 \mathrm{~g}$ pro Woche; die Gewichtskurve nimmt während dieser Zeit einen sanft ansteigenden, durch mäßige Schwankungen unterbrochenen Verlauf. Körperlänge nimmt in derselben Zeit (10 Wochen) trotz des mangelhaften Gewichtsanstiegs von $58^{3} / 4 \mathrm{~cm}$

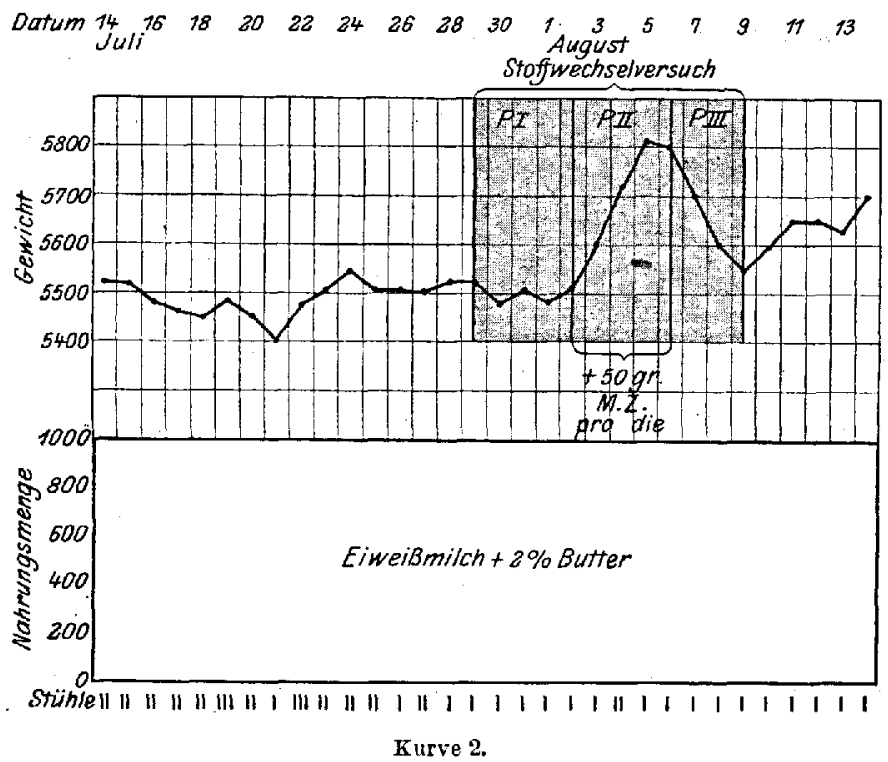

bis auf $631 / 2 \mathrm{~cm} \mathrm{zu}$, d. h. um $4 \frac{3}{4} \mathrm{~cm}$. Das Längenwachsțum beträgt demnach erheblich mehr, als dem Gewichtszuwachs entspricht (analog dem Verhalten bei einfacher Unterernährung). Keine Erscheinungen von Rachitis.

Vom 14. VII. an, wo unsere Kurve einsetzt, erhält das Kind $1000 \mathrm{~g}$ Eiweißmilch mit 2\% Butterzusatz. Der bestimmte Gehalt der Nahrung an Fett beträgt $43 \mathrm{~g}$, an Eiweiß 35,2 g. Der Zuckergehalt wurde nicht bestimmt, dürfte aber auf etwa $15 \mathrm{~g}$ (Durchschnittswert des Gehalts der Eiweißmilch) zu veranschlagen sein. Die zugeführte Nahrung enthält danach 605 Calorien, was bei einem Gewicht von $5500 \mathrm{~g}$ des Kindes etwa 110 Calorien pro Kilogramm entspricht. Damit dürfte der Energiebedarf gedeckt sein. 
Zur Wirkung der Kohlehydrate anf den Anwuchs im Säuglingsalter. 343

Der Stoffwechselversuch gliedert sich; wie die Kurve II zeigt, in 3 Perioden.

$$
\begin{aligned}
& \text { Periode I: 29. VII.-2. VIII. (4 Tage). } \\
& \text { Periode II: 2. VIII.-6. VIII. (4 Tage). } \\
& \text { Periode III: 6. VIII.-9. VIII. (3 'Tage). }
\end{aligned}
$$

Die Grundnahrung war in allen drei Perioden dieselbe. In Periode II Zulage von $50 \mathrm{~g}$ Milchzucker pro die. Uber den Gewichtsverlanf siehe Kurven.

Entleerungen waren während der ganzen Versuchsclauer von gleicher fester Konsistenz und hellgelber Farbe.

Das Kind lag ruhig auf der Schwebe, trank alle Nahrung, kein einziges Mal Erbrechen.

In den ersten Tagen des Versuchs starkes Schwitzen.

Ich lasse die Resultate in Form von Tabellen, welche die erhaltenen

\begin{tabular}{|c|c|c|c|}
\hline Periode & Nahrung & Einfuhr & $\begin{array}{l}\text { Neutralfett } \\
+ \text { Fettsäuren } \\
\text { im Bot }\end{array}$ \\
\hline$\cdot \mathrm{J}$ & $1000 \mathrm{~g}$ Eiweißmilch $72 \%$ Butter & 43,00 & 0,47 \\
\hline II & $\begin{array}{c}1000 \text { g Eiweilmilch }+2 \% \text { Butter } \\
+50 \text { g Milchzucker }\end{array}$ & 43,00 & 0,64 \\
\hline III & $1000 \mathrm{~g}$ Eiweißmilch $+2 \%$ Butter & 43,00 & 0,66 \\
\hline
\end{tabular}
Durchschnittswerte pro die erhalten, folgen.

Fettbilanz (pro die).

Von dem Fettstoffwechsel wurde nur Einfuhr und die Ausfuhr von Neutralfett plus Fettsäuren bestimmt. Wie im vorigen Versuche erscheint die Fettausnutzung eine vorzügliche, allerdings ist in der Periode der Zuckerzulage, ähnlich wie in Vorsuch I und in der nächstfolgenden eine geringe Erhöhung der Neutralfettausscheidung zn kon-

\begin{tabular}{|c|c|c|c|c|c|c|c|}
\hline $\mathrm{Pe}$ & \multirow{2}{*}{ Nahrung } & \multirow{2}{*}{ Einfuhr } & \multicolumn{2}{|c|}{$\begin{array}{l}\text { Kot } \\
\text { - in } \% \text { der }\end{array}$} & \multirow{2}{*}{ Urin } & \multicolumn{2}{|c|}{ Retention } \\
\hline riade & & & absolut & in \% der & & absolut & $\begin{array}{l}\text { in \% der } \\
\text { Einfuhr }\end{array}$ \\
\hline I & $1000 \mathrm{~g}$ Eiweißmilch $+2 \%$ Butter & 5,63 & 0,262 & 4,65 & 4,523 & $+0,845$ & 15,01 \\
\hline II & $\begin{array}{c}1000 \mathrm{~g} \text { Eiweißmilch }+2 \% \text { Butter } \\
+50 \mathrm{~g} \text { Milchzucker }\end{array}$ & 5,63 & 0,317 & 5,63 & 3,708 & $+1,605$ & 28,51 \\
\hline IIII & $1000 \mathrm{~g}$ Eiweißmilch $+2 \%$ Butter & 5,63 & 0,307 & 5,45 & 4,568 & $+0,755$ & 13,41 \\
\hline
\end{tabular}
statieren.

$$
\text { N-Bilanz (pro die). }
$$

Die N-Bilanz verhält sich hier anders wie im vorigen Versuch. Anch bei der zuckerarmen Kost eine gute Retention, die allerdings durch die 
Zuckerzulage noch erheblich steigt. Gewicht und $\mathrm{N}$-Retention gehen nicht völlig parallel (Poriode I, gute N-Retention bei Gewichtsstillstand).

$\mathrm{K}_{2} \mathrm{O}$-Bilanz (pro die).

\begin{tabular}{|c|c|c|c|c|c|c|c|}
\hline $\begin{array}{c}\text { Pe- } \\
\text { riode }\end{array}$ & Nahrung & Finfuhr & absolut & $\begin{array}{l}\text { Kot } \\
\text { in \% der } \\
\text { Einfuhr }\end{array}$ & Uurin & $\begin{array}{r}\text { Reten } \\
\text { absolut }\end{array}$ & $\begin{array}{l}\text { ntion } \\
\text { in \% der } \\
\text { Kinfuhr }\end{array}$ \\
\hline \multirow[t]{2}{*}{ I } & \multirow{2}{*}{$\begin{array}{l}1000 \mathrm{~g} \text { Eiweißmilch }+2 \% \text { Butter } \\
1000 \mathrm{~g} \text { Eiweißmilch }+2 \% \text { Butter }\end{array}$} & \multirow{2}{*}{$\begin{array}{l}1,011 \\
1,011\end{array}$} & 0,113 & $11,18^{\circ}$ & 0,609 & $+0,289$ & 28,59 \\
\hline & & & 0,125 & 12,36 & 0,413 & $+0,473$ & 46,79 \\
\hline HI & $\begin{array}{c}+50 \mathrm{~g} \mathrm{Milchzucker} \\
1000 \mathrm{~g} \text { Eiweißmilch }+2 \% \text { Butter }\end{array}$ & 1,011 & 0,161 & 15,92 & 0,730 & $+0,120$ & 11,87 \\
\hline III & \multicolumn{7}{|c|}{$\mathrm{Na}_{2} \mathrm{O}$-Bilanz (pro die). } \\
\hline $\begin{array}{c}\text { Pe- } \\
\text { riode }\end{array}$ & Nahrung & Einfuhr : & \multicolumn{2}{|c|}{ Kot $\begin{array}{l}\text { in \% der } \\
\text { Einfuhr }\end{array}$} & Urin & $\begin{array}{r}\text { Reten } \\
\text { absolut }\end{array}$ & $\begin{array}{l}\text { nion } \\
\text { in \% der } \\
\text { Enfinur }\end{array}$ \\
\hline \multirow[t]{2}{*}{ I } & $1000 \mathrm{~g}$ & 0,477 & 0,042 & 1 & 0,263 & $+0,1 \%$ & 36,06 \\
\hline & $1000 \mathrm{~g}$ Eiweißmilch $+2 \%$ Butter & 0,477 & 0,082 & 17,19 & 0,100 & $+0,295$ & 61,84 \\
\hline 11 & $\mathrm{Mi}$ & & & & & & \\
\hline $\mathrm{III}$ & $1000 \mathrm{~g}$ Eiweißmilch $+2^{0}{ }_{10}$ Butter & 0,477 & 0,086 & 18,03 & 0,233 & $+0,158$ & 33,12 \\
\hline
\end{tabular}

Die $\mathrm{K}_{2} \mathrm{O}$ und $\mathrm{Na}_{2} \mathrm{O}-$ Bilanz verhalten sich ganz ähnlich der $\mathrm{N}$-Bilanz. $\mathrm{Cl}(\mathrm{NaCl})-\mathrm{Bilanz}$ (pro die).

\begin{tabular}{|c|c|c|c|c|c|c|c|}
\hline $\begin{array}{c}\text { Pe- } \\
\text { riode } \\
2\end{array}$ & Nahrung & Einfuhr & absolut & $\begin{array}{l}\text { Kot } \\
\text { in \% der } \\
\text { Einfuhr }\end{array}$ & Urin & $\begin{array}{r}\text { Reter } \\
\text { absolut }\end{array}$ & $\begin{array}{l}\text { in \% der } \\
\text { Einfuhr }\end{array}$ \\
\hline I & $\begin{array}{c}1000 \mathrm{~g} \text { Eiweibmilch }+20 \mathrm{~g} \\
\text { Butter }\end{array}$ & 0,467 & 0,033 & 7,07 & 0,515 & 81 & - \\
\hline II & $\begin{array}{l}1000 \mathrm{~g} \text { Eiweißmilch }+20 \mathrm{~g} \\
\text { Butter }+50 \mathrm{~g} \mathrm{Milchzucker}\end{array}$ & 0,467 & 0,023 & 4,92 & 0,405 & $+0,039$ & 8,35 \\
\hline III & $\begin{array}{c}1000 \mathrm{~g} \text { Eiwoißmilch }+20 \mathrm{~g} \\
\text { Butter }\end{array}$ & 0,467 & 0,027 & 5.78 & 0,858 & $-0,418$ & - \\
\hline
\end{tabular}

Auffallend ist im Gegensatz dazu die schlechte Cl-Bilanz. In Periode I leicht negativ wird sie durch die Zuckerzulage schwach positiv, um mit der Zuckerentziehung eine erhebliche Verschlechterung zu erfahren.

CaÓ-Bilanz (pro die).

\begin{tabular}{|c|c|c|c|c|c|c|c|}
\hline $\begin{array}{c}\mathrm{Pe}- \\
\text { riode }\end{array}$ & Nahrung & Einfuhr & $\begin{array}{r}\text { K } \\
\text { absolut }\end{array}$ & $\begin{array}{l}\text { Zot } \\
\text { in \%o der } \\
\text { Einfuhr }\end{array}$ & Urin & $\begin{array}{r}\text { Reten } \\
\text { absolut }\end{array}$ & $\begin{array}{l}\text { ntion } \\
\text { is } \% \text { der } \\
\text { Einfulur }\end{array}$ \\
\hline I & 1000 g Eiweißmilch $+2 \%$ Butter & 0,996 & 0,878 & 88,16 & & $+0,118$ & 11,84 \\
\hline II & $\begin{array}{l}1000 \mathrm{~g} \text { EiweiBmilch }+2 \% \text { Butter } \\
+50 \mathrm{~g} \mathrm{Milchzucker}\end{array}$ & 0,996 & 0,871 & 87,45 & & $+0,125$ & 12,55 \\
\hline III & $1000 \mathrm{~g}$ Eiweikmilch +2 \% Butter & 0,996 & 0,968 & 97,19 & & $+0,028$ & 2,81 \\
\hline
\end{tabular}


Die Kalkbilanz verhält sich ähnlich wie in Versuch I, keine Beeinflussung durch Zuckerzulage, wohl aber Verschlechterung bei plötzlicher Entzjehung des vorher verabreichten Zuckers.

$\mathrm{P}_{2} \mathrm{O}_{5}-$ Bilanz (jio die).

\begin{tabular}{|c|c|c|c|c|c|c|c|}
\hline $\begin{array}{c}\text { Pe- } \\
\text { riode }\end{array}$ & Nahrung & Einfuhr & $\begin{array}{r}\mathrm{K} \\
\text { absolut }\end{array}$ & $\begin{array}{l}\text { ot } \\
\text { in \% der } \\
\text { Einfuhr }\end{array} \mid$ & Urin & $\begin{array}{r}\text { Reten } \\
\text { absolut }\end{array}$ & $\begin{array}{l}\text { ntion } \\
\text { in \% der } \\
\text { Einfuhr }\end{array}$ \\
\hline I & $1000 \mathrm{~g}$ Eiweißmilch $+2 \%$ Butter & 1,479 & 0,503 & 34,01 & 0,783 & $+0,193$ & 13,05 \\
\hline II & $\begin{array}{c}1000 \mathrm{~g} \text { Eiweißmilch }+2 \% / 0 \text { Butter } \\
+50 \mathrm{~g} \mathrm{Milch} \text {.ucker }\end{array}$ & 1,479 & 0,393 & 26,57 & 0,616 & $+0,470$ & 31,78 \\
\hline III & $1000 \mathrm{~g}$ Eiweißmilch $+2^{0}:{ }_{0}$ Butter & 1,479 & 0,537 & 36,31 & 0,807 & $+0,135$ & 9,13 \\
\hline
\end{tabular}

Die Phosphorretention gestaltet sich im Gegensatz zu den vorhergehenden Versuchen unter Zuckerzulage weit günstiger. Es folgt nun eine Ubersicht über das Verhalten der einzelnen Bilanzen beim Kind K. R.

Kind R. Bilanz pro die.

\begin{tabular}{|c|c|c|c|c|c|c|c|}
\hline Periode & Nabrung & $\mathbf{N}$ & $\mathrm{Cl}(\mathrm{NaCl})$ & $\mathrm{Na}_{\mathrm{s}} \mathrm{O}$ & $\mathbf{K}_{2} \mathrm{O}$ & $\mathrm{CaO}$ & $\mathrm{P}_{2} \mathrm{O}_{6}$ \\
\hline $\mathrm{I}$ & $\begin{array}{l}840 \mathrm{~g} \text { Eiweißmilch } \\
\quad+2 \% \text { Butter }\end{array}$ & $-0,102$ & $-0,075$ & $+0,067$ & $-0,042$ & $+0,502$ & $+0,337$ \\
\hline II & $\begin{array}{c}840 \mathrm{~g} \text { Eiweißmilch } \\
+2 \% \text { Butter }+40 \mathrm{~g} \\
\quad \text { Milchzucker }\end{array}$ & $+0,411$ & $+0,060$ & $+0,189$ & $+0,106$ & $+0,491$ & $+0,234$ \\
\hline III & do. & $+0,484$ & $+0,020$ & $+0,114$ & $+0,120$ & $+0,522$ & $+0,330$ \\
\hline IV & $\begin{array}{c}840 \mathrm{~g} \text { Eiweißmilch } \\
+2 \% \text { Butter }\end{array}$ & $-0,038$ & $-0,513$ & $-0,160$ & $-0,086$ & $+0,368$ & $+0,036$ \\
\hline $\mathrm{V}$ & do. & $-0,128$ & $-0,082$ & $+0,102$ & $+0,113$ & $+0,459$ & $+0,392$ \\
\hline
\end{tabular}

Die Vorperiode ist charakterisiert durch eine negative $\mathrm{N}, \mathrm{K}_{2} \mathrm{OCl}$ Bilanz, die $\mathrm{Na}_{2} \mathrm{O}$-Bilanz ist leicht positiv. Die $\mathrm{CaO}+\mathrm{P}_{2} \mathrm{O}_{5}$-Bilanz erscheinen dahingegen normal. Durch die Zuckerzufuhr erfährt die $\mathrm{N}$ $\mathrm{Na}_{2} \mathrm{O}, \mathrm{K}_{2} \mathrm{O}$ und Cl-Bilanz eine erhebliche Verbesserung, während die Ca und $\mathrm{P}_{2} \mathrm{O}_{5}$-Bilanz unverändert bleiben. Zuckerentziehung verschlechtert $\mathrm{N}, \mathrm{Cl}, \mathrm{Na}_{2} \mathrm{O}$ und $\mathrm{K}_{2} \mathrm{O}$-Bilanz wieder erheblich, führt aber auch zu einer vorübergehenden, leichten Vermehrung der Kalk- und einer stärkeren der Phosphorausscheidung.

Bezüglich des Verhaltens der Stickstoff-, Chlor- und Alkalibilanz zum Gewicht ist im allgemeinen eine Parallele vorhanden, doch finden im einzelnen Ausnahmen statt. 
Kind W. Gesamtbilanz pro die.

\begin{tabular}{|c|c|c|c|c|c|c|c|}
\hline Periode & Nahrung & $\mathrm{N}$ & $\mathrm{Cl}(\mathrm{NaCl})$ & $\mathrm{K}_{2} \mathrm{O}$ & $\mathrm{Na}_{2} \mathrm{O}$ & $\mathrm{P}_{8} \mathrm{O}_{3}$ & $\mathrm{CaO}$ \\
\hline I & $\begin{array}{l}1000 \mathrm{~g} \text { Eiweißmilch } \\
+2 \% \text { Butter }\end{array}$ & $+0,845$ & $-0,081$ & $+0,289$ & $+0,172$ & $+0,193$ & $+0,118$ \\
\hline II & $\begin{array}{c}1000 \mathrm{~g} \text { Eiweißmilch } \\
+2 \% \text { Butter }+50 \mathrm{~g} \\
\text { Rohrzucker }\end{array}$ & $+1,605$ & $+0,039$ & $+0,473$ & $+0,295$ & $+0,470$ & $+0,125$ \\
\hline III & $\begin{array}{l}1000 \mathrm{~g} \text { EiweiBmilch } \\
+2 \% \text { Zucker }\end{array}$ & $+0,755$ & $-0,418$ & $+0,120$ & $+0,158$. & $+0,135$ & $+0,028$ \\
\hline
\end{tabular}

Die Übersicht übcr die Gesamtbilanz des Kindes W. ergibt in den zuckerarmen Perioden annähernd normale Retentionswerte. Nur die Chlorbilanz erscheint leicht negativ. Durch die Zuckerzufuhr werden N, $\mathrm{Cl}, \mathrm{K}_{2} \mathrm{O}$ und $\mathrm{Na}_{2} \mathrm{O}-\mathrm{Bilanz}$ erheblich gebessert, aber auch die $\mathrm{P}_{2} \mathrm{O}_{5}-\mathrm{Bi}$ lanz. Bezüglich des Kalks ist unter der Zuckerzufuhr keine Veränderung zu konstatieren, wohl aber ein vorübergehendes Sinken der Kalkretention während der Zuckerentziehung.

Den beiden eben aufgeführten Stoffwechselbilanzen ist gemeinsam das Verhalten in der Vorperiode, die Wirkung der Zuckerzulage auf $\mathrm{N}$, $\mathrm{Cl} ; \mathrm{K}_{2} \mathrm{O}$ und $\mathrm{Na}_{2} \mathrm{O}$-Stoffwechsel und das Fehlen der Wirkung auf den $\mathrm{Ca}$-Stoffwechsel. Weiterhin das bis zu einem gewissen Grade parallele Verhalten von Gewicht und Retention von Stickstoff und Chloralkalien, das allerdings, wie schon erwähnt, vielfach Ausnahmen erkennen läßt, besonders dann, wenn es sich um Gewichtsstillstand oder Abnahme handelt. Mit der Wirkung auf die N- und Cl-Bilanz werden.die bisher vorliegenden Resultate über den Finfluß des Zuckers bestätigt. Das gleiche gilt von dem längst vermuteten Zusammenhang zwischen Kohlehydrat und Natrium- bzw. Kaliumretention.

Dis Fehlen der Wirkung der Zuckerzufuhr auf die Kalkretention beim Gesunden bestätigt den von Freund für die Malzwirkung erhobenen Befund und steht allerdings in einem gewissen Widerspruch zu den früher erwähnten Resultaten von Kochmann und Birk-Rothberg. Auffallend ist bezzüglich der Kalkbilanz die in beiden Versuchen feststellbare leichte Verringerung der Retentionswerte bei der Zuckerentziehung.

Differenzen bezüglich der beiden Stoffwechselbilanzen bestehen besonders hinsichtlich der Resultate in den Perioden der zuckerarmen Ernährung. Bei Kind K. R. erscheinen die Bilanzen des Stickstoffes, der Alkalien und des Chlors in den Perioden zuckerarmer Ernährung im allgemeinen negativ, bei Kind W. positiv. Vielleicht ist diese Differenz 
Zur Wirkung der Kohlehydrate auf den Anwuchs im Săuglingsalter. $\quad 347$

darauf zurückzuführen, daß bei Kind W. dem Versuche eine wochenlange zuckerarme Ernährung vorhergegangen und damit vielleicht eine gewisse Anpassung zustande gekommen war. Wie das differente Verhalten des Phosphors zu erklären ist, wage ich nicht zu entscheiden.

Die vorliegenden Untersuchungen ergeben also im wesentlichen eine Bestätigung der bekannten spezifischen Wirkung des Zuckers für eine normale Wasser-, Alkali-, Chlor- und Stickstoffretention; sie zeigen, daß bei einer mangelhaften Zuckerzufuhr auch dann, wenn der Calorienbedarf gedeckt ist und alle sonstigen Stoffe in genügender Menge vorhanden sind, die $\mathrm{N}-, \mathrm{Cl}-, \mathrm{Na}_{2} \mathrm{O}-, \mathrm{K}_{2} \mathrm{O}$-Bilanz sogar stark negativ werden kann, während die Ca-Bildung und $\mathrm{P}_{2} \mathrm{O}_{5}$-Bilanz keine Einbuße zu erleiden brauchen; sie zeigen ferner, daß die vorher negativen Bilanzen bei Zuckerzulagen in positive umschlagen.

Soweit der spezifische Effekt des Zuckers, den man nach allen klinischen Erfahrungen wohl von dem in unseren Versuchen verwendeten Milchzucker auf die anderen Zuckerarten übertragen kann, im Stoffwechsel.

Es ergibt sich nun eine weitere sehr wichtige Frage für das Verständnis der Zuckerwirkung. Besteht hier nur ein Nebeneinander von Stoffwechselwirkungen oder ist ein Abhängigkeitsverhältnis vorhanden? Für ein solches spricht die im allgemeinen zu beobachtende Parallele in dem Verhalten von Stickstoff, Wasser und Alkalichloriden bei Zuckerzulage, gegen ein solches die nicht selten beobachteten Abweichungen von dem Parallelismus, in qualitativer, vor allem aber in quantitativer Hinsicht.

Klinische Erfahrungen haben uns im Laufe der Jahre zu der Uberzeugung gebracht, daß ein solches Abhängigkeitsverhältnis der beobachteten Stoffwechselwirkungen vorliegt.

Man kann beobachten, daß die Zuckerwirkung abhängig ist von der Menge gerade derjenigen Stoffe in der Nahrung, die auch im Stoffwechsel in engem Zusammenhang mit der Zuckerwirkung zu stehen scheinen, d. h. dem Wasser, den wasserretinierenden "Salzen und dem. Eiweiß. Sehon grobklinische Erfahrungen demonstrieren, daß der Zucker da am meisten gewichtsbefördernd wirkt, wo auch hohe Salzund Eiweißmengen in der Nahrung vorhanden sind (Buttermilch), und $\mathrm{da} B$ die Zuckerwirkung um so geringer wird, je geringer der Salzund Eiweißgehalt der Nahrung ( $1 / 3$ Milch und Frauenmilch).

Mit Sicherheit läßt sich die Abhängigkeit der Zuckerwirkung von dem vorhandenen Salz- und Eiweißgehalt der Nahrung erweisen, wenn man den Zucker ciner Nahrung zulegt, in der einer dieser beiden 
Stoffe fehlt. Ich führe hier zunächst die Kurve eines Kindes an, das als Nahrung vorübergehend eine salzfreie Kost erhielt, in Form von in destilliertem Wasser aufgelöstem Käse (Kurve 3). Man'sieht, daß die Zuckerzulage hier gänzlich ohne Effekt ist.

Ähnliche Beobachtungen haben wir bei der Zuckerzulage zu einer eiweißfreien Nahrung gewonnen, doch erscheinen die diesbezüglichen Beobachtungen aus dem Grunde noch nicht beweisend, weil hier eine

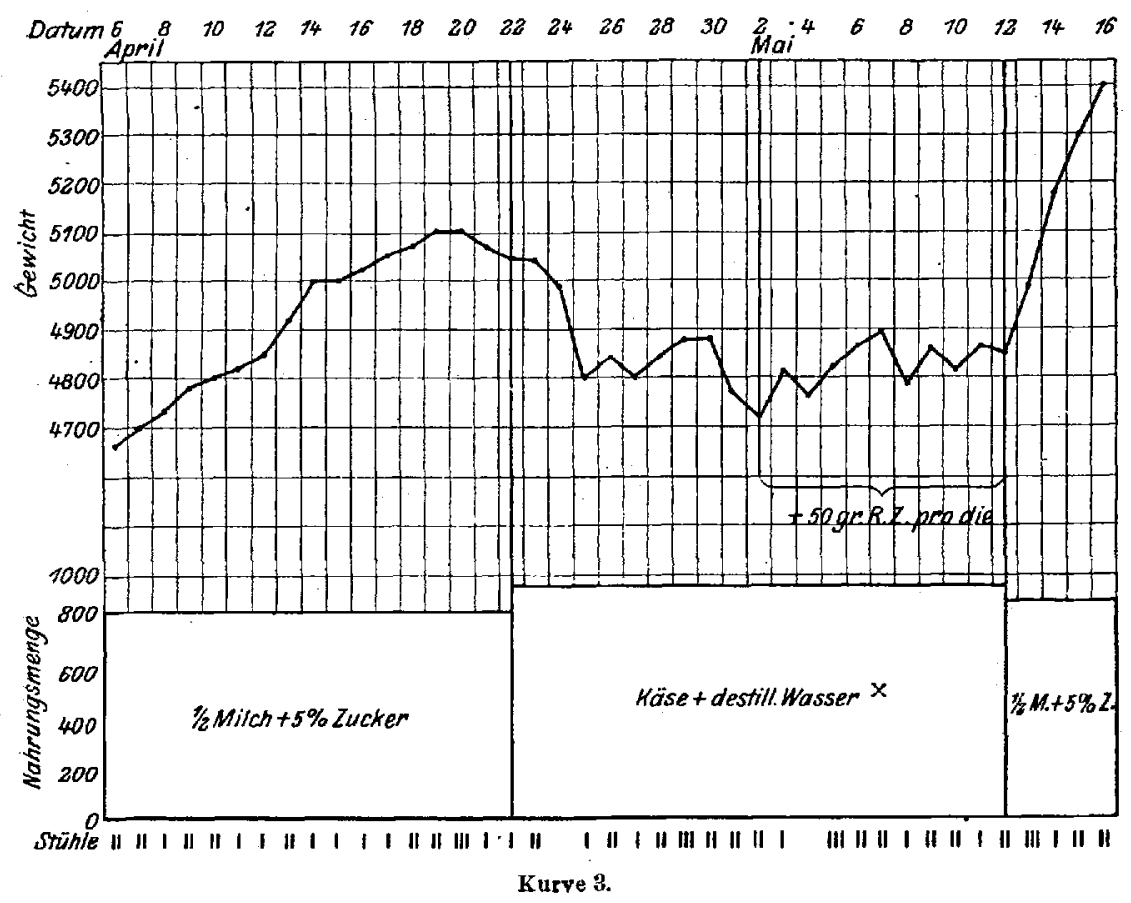

Vorbedingung zur Anstellung dieser Untersuchungen nicht erfüllt war: die Entleerungen waren nicht normal, sondern leicht dyspeptisch.

- Immerhin lassen solche Beobachtungen den Schluß zu, daß die gewichtssteigernde, d. h. im wesentlichen die wasserretinierende Wirkung des Zuckers an das Vorhandensein anderweitiger Nährstoffe gebunden, zum mindesten aber in erheblichem Maße von diesen abhängig ist. Es scheint, als ob der Zuckerzusammen mit dem Wasser, den Alkalichloriden und dem Eiweiß einen Ring von Nährstoffen bildet, die den Wasseransatz beherrschen, und zwar so, daß durch das Kohlehydrat das Wasser und mit ihm die gelösten Salze und N-haltige Substanz zurückgehalten werden. 
Die Zusammengehörigkeit der hier genannten Stoffe und ihre Bedeutung für die Wasserretention erweist sich denn auch noch durch anderweitige klinische Beobachtungen, die ergeben haben, daß i m mer dann, wenn ein Glied in dem Ring fehlt, d. h. wenn einer dergenannten Nährstoffe in nicht genügender Menge in der Nahrung vorhanden ist, eine Gewichtsvermehrung nicht möglich ist, und daß die Zulage geringer Quantitäten des betreffenden Nährstoffes genügt, um einen steilen Gewichtsansatz zu crzielen, der in gar keinem Verhältnis zu der Menge des dargereichten $S$ toffes steht. Die folgenden Beobachtungen mögen dies beweisen:

1. Wasserarme Ernährung (Kurve 4). Ich nehme die hier vorliegende Kurve einer Arbeit von L. F. Me yer ${ }^{\mathbf{1}}$ ).

Trotz kalorisch ausreichender Ernährung nimmt das Kind, offenbar infolge $\mathrm{H}_{2} \mathrm{O}$ - Mangels, bei der konzentrierten Eiweiß-

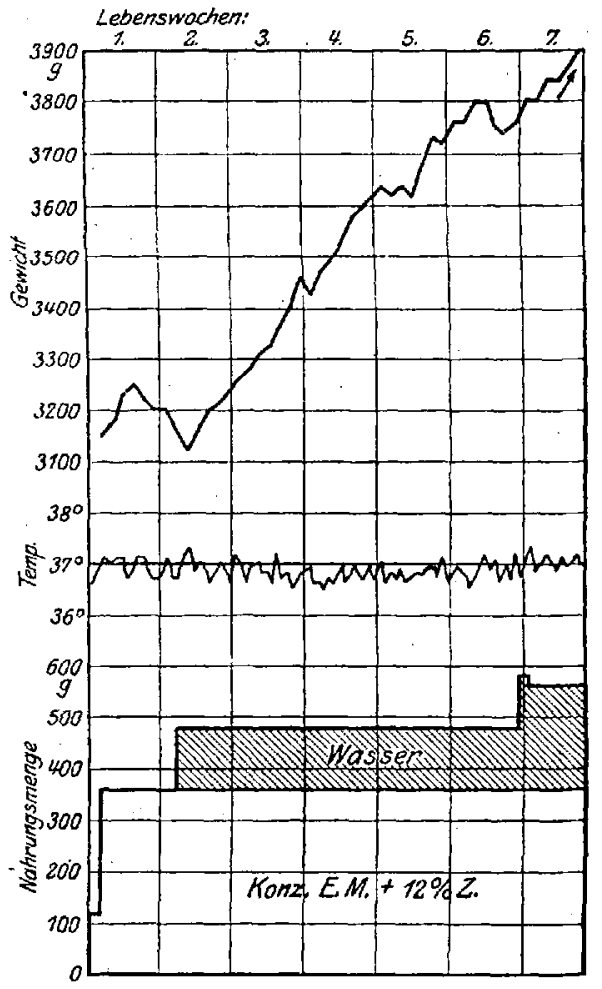

Kurve 4. Gewichtsstillstand unter Schwankungen b el Ennährung mit konzentrierter EïweiBmilch, Zunahme bei Wasserzulage.

milch nicht zu. Bèi Zulage von ca. $100 \mathrm{~g}$ Wașser pro die erfolgt steiler Gewichtsanstieg.

2. Zuckerarme Ernährung (Kurve 5) Die Kurve ist früheren Arbeiten von $\mathrm{mir}^{2}$ ) entnommen.

Trotz außerordentlich calorienreicher Ernährung erfolgt bei dem 5 Monate alten Kinde keine Gewichtszunahme. Erst auf Zuckerzusatz Umbiegen der Kurve zu steilem Gewichtsanstieg. Es genügen $5 \mathrm{~g}$ pro

j) L. F. Meyer, Zeitschr. f. Kinderheilk. 5.

2) Rosenstern, Zeitschr. f. Kinderheilk. 2.

Zeitschrift für Kinderhellkunde. 0 . XVIII. 
die, um (erste Periode der Zunahme) innerhalb 7 Tagen einen Ansatz. von $420 \mathrm{~g}, \mathrm{~d} . \mathrm{h} .60 \mathrm{~g}$ pro die zu erzielen.

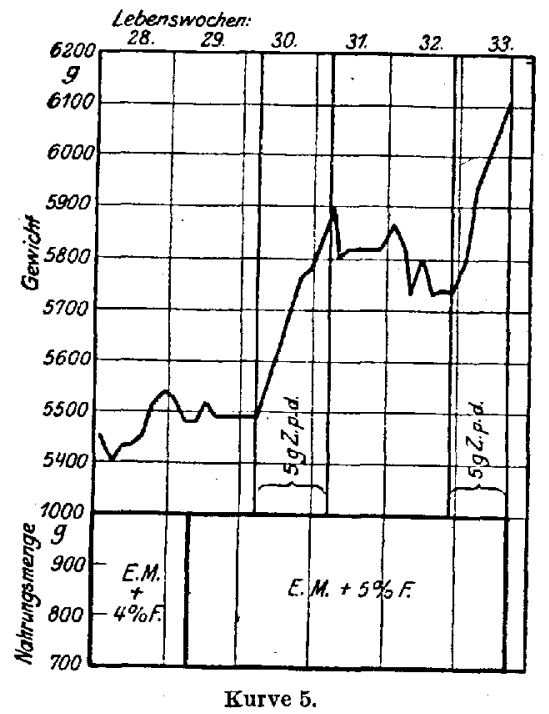

3. Salzarme Ernährung (Kurve 6). Bei $1 / 2 \mathrm{~N}$ mit Zucker zeigt das 3 Monate alte Kind befriedigende Gewichtszunahme. Ubergang auf Käse + Zucker (Käse aus 11 Vollmilch $+50 \mathrm{~g}$ Zucker in $800 \mathrm{~g}$ destilliertem Wasser) läßt die Gewichtskurre nach vorübergehendem Abfall horizontal verlaufen. Ersatz von $100 \mathrm{~g}$ Wasser durch Molke pro die genügen, um einen steilen Aufschwung der Gewichtskurve zu erzeugen.

4. Eiweißarme Ernährung (Kurve 7). Das 1 Monat alte Kind erhält żunächst eine Nahrung, die im Liter $250 \mathrm{~g}$ Milch, $100 \mathrm{~g} 30$ proz.

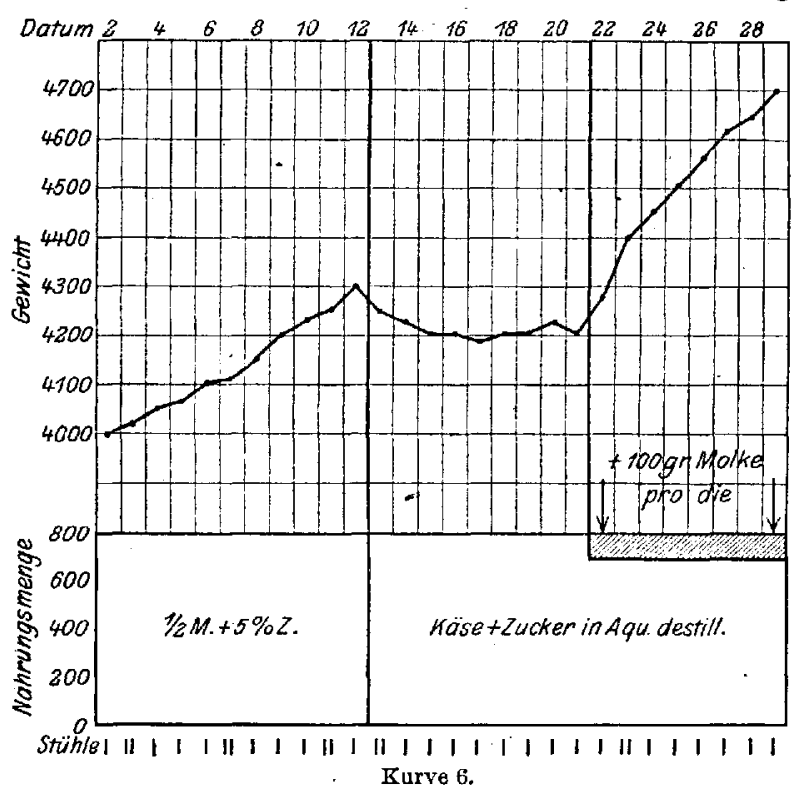

Casein, $50 \mathrm{~g}$ Zucker und $650 \mathrm{~g}$ Wasser enthält. Der Eiweißgehalt dieser Nahrung ist gering (entspricht noch nicht dem der Frauenmilch). $5 \mathrm{~g}$ Eiweiß (Albulactin $=$ Ca-Albuminat) pro die bewirken innerhallb 5 Tagen einen Gewichtsanstieg von 2910 auf $3230 \mathrm{~g}$, d. h. $320 \mathrm{~g}$ Ansatz). Nun istallerdings die wasserbindende Wirkung des Eiweißes hier nicht so einwandfrei sichergestellt wie etwa die des Zuckers, da die Stühle - während der 
Znr Wirkung del Kohlehydrate auf den Anwuchs in Siuglingsalter. 351

ganzen Versuchsdauer - leicht dyspeptisch waren und damit anderweitige Wirkungen mitspielen könnten.

Unter Vorbehalt dieser Einschränkung erscheint uns durch die hier mitgeteilten klinischen Erfahrungen der oben ausgesprochene Satz gestützt: daß Kohlehydrat, Wasser, Alkalichloride und Eiweiß einen Ring von Nährstoffen bilden, die den Wasseransatz beherrschen, derart, daß immer dann, wenn einer der genaunten Stoffe in zu geringer Menge in der Nahrung vorhanden ist, eine Gewichtsvermehrung nicht möglich

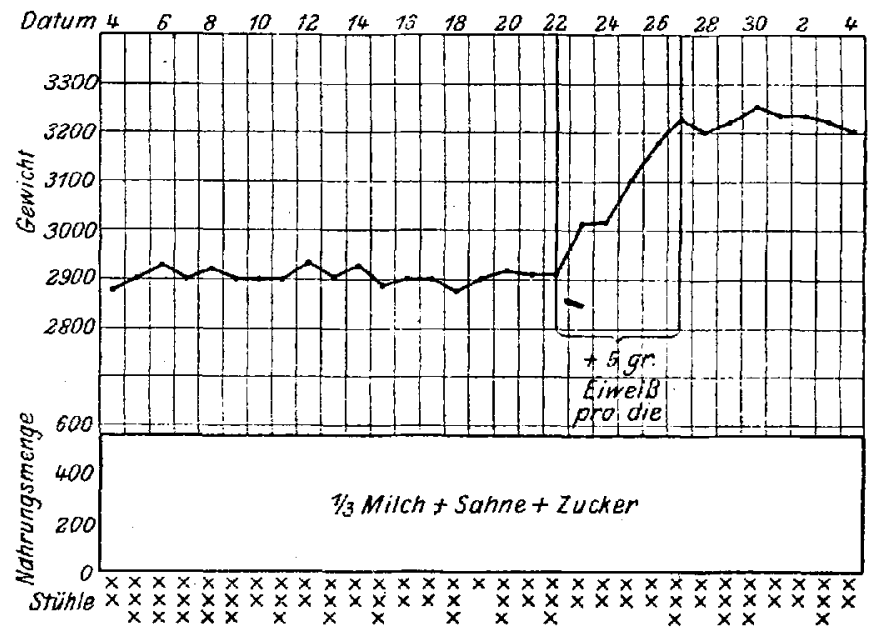

Kurve 7.

ist, und daß die Zulage geringer Quantitäten des betr. Nährstoffs genügt, um einen ateilen Gewichtsansatz zu erzielen, der nur durch $\mathrm{H}_{2} \mathrm{O}$ Retention erklärt werden kann und damit dic wichtigste Vorbedingung für das Wachstum, die Quellung des Gewebes, zu cxfüllen.

Im Gegensatz zu den Wirkungen der genannten' Stoffe steht das Verhalten von Fott und Kalk, denen ein derartiger Einfluß auf die $\mathrm{H}_{2} \mathrm{O}$-Retention und damit auf das Gewebswachstum offenbar fehlt.

Innerhalb des Konzerns der für die Wasserbindung bzw. das Gewebswachstum in Frage kommenden Stoffe spielt das Kohlehydrat eine führende Rolle. Wie man sich seine Wirkung vorzustellen hat, ist fraglich. Finkelstein ${ }^{1}$ ) ist der Ansicht, „da B aus dem Kohlehydrat im Verein mit dem Eiweiß eine quellbare kolloidale Substanz

1) Finkelstein, Zeitschr. f. ärztl. Fortbildung 1914, Nr. 4. 
cntsteht, die zunächst Salze in sich aufnimmt, und daß diese wieder Wasser"anziehen, welche das Kolloid zum Quellen bringen... Wo diese Wirkung stattfindet, entzieht sich unserer Kenntnis. Wir wissen nur so viel, daß normale Darmfunktionen die Voraussetzung für die Bindung der genannten Substanzen bilden. Völlig unbekannt ist uns,

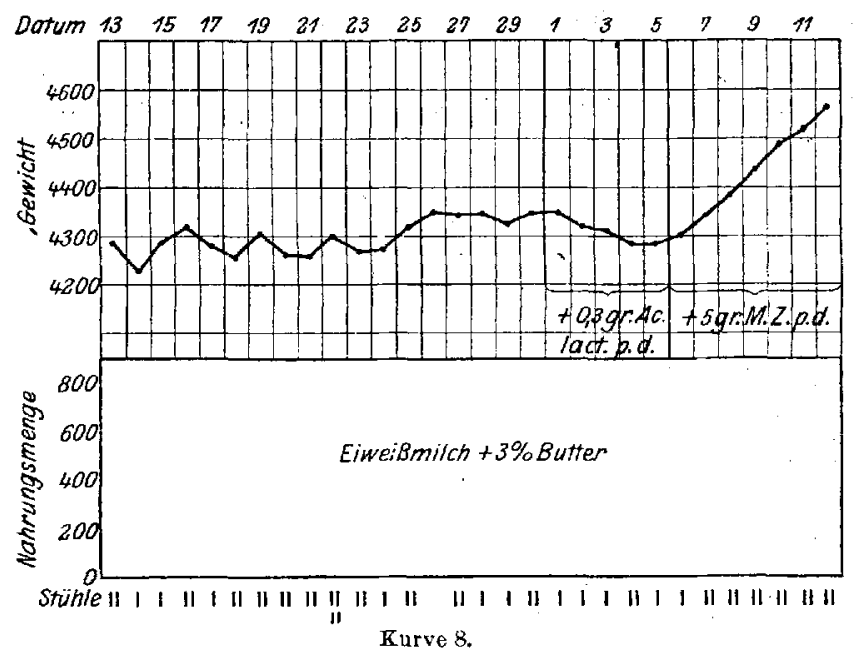

weiter, ob dem Zucker selbst oder irgendwelchen Abbauprodukten die Wirkung zuzúschreiben jst. Man könnte am ehesten noch an den Effekt gewisser aus dem Zucker gebildeter Säuren denken, deren Wirkung auf den Salz- und Stickstoffstoffwechsel durch Klotz sichergestellt ist. Aber wie die vorstehende Kurve 8 zeigt, ist es uns bis jetzt nicht gelungen, die Wirkung des Zuckers durch Zufuhr kleiner Säuremengen $z u$ ersetzen. 